\title{
CARACTERIZACIÓN, MEDIANTE ESPECTROMETRÍA DE MASAS DE ALTA RESOLUCIÓN MALDI/FT-ICR, DE TANINOS HIDROLIZABLES DE LA TARA \\ (Caesalpinia spinosa)
}

\author{
Juan Z. Dávalosª, Violeta L. Romero ${ }^{\text {b }}$, Juan I. Sánchez ${ }^{\mathrm{c}}$, Julissa Chávez ${ }^{\mathrm{d}}$, \\ Ana Valderrama-Negrón ${ }^{\mathrm{d}}$
}

\begin{abstract}
RESUMEN
Estudiamos y caracterizamos la estructura de taninos hidrolizables de tara comercial en polvo, mediante espectrometría de masas de ultra-alta resolución (MALDI/FT-ICR) sin empleo de técnicas de separación. Nuestros resultados experimentales, fundamentados por cálculos computacionales al nivel DFT/M05-2X, muestran que las especies identificadas son básicamente estructuras oligoméricas de ácidos poligaloquínicos del tipo $\left(\mathrm{C}_{7} \mathrm{H}_{4} \mathrm{O}_{4}\right)_{\mathrm{n}}\left(\mathrm{C}_{7} \mathrm{H}_{12} \mathrm{O}_{6}\right)$ formados por una cadena de residuos de ésteres gálicos (dépsido) unida, vía enlace de éster, a un ácido quínico.
\end{abstract}

Palabras clave: Caesalpinia spinosa, tara comercial en polvo, taninos hidrolizables, MALDI/ FT-ICR, teoría DFT/M05-2X, ácidos poligaloquínicos, dépsidos gálicos.

\section{CHARACTERIZATION, BY HIGH RESOLUTION MALDI/FT- ICR MASS SPECTROMETRY, OF TARA (Caesalpinia Spinosa) HYDROLYSABLE TANNINS}

\begin{abstract}
We have studied and characterized the structure of hydrolysable tannins of commercial powder tara, performed by means ultra-high-resolution mass spectrometry (MALDI/FT-ICR) without using separation techniques. Our experimental results, supported by computational calculations at DFT/ M05-2X level of theory, show that the identified species are basically oligomeric structures of polygalloyquinic acids, of the (C7H4O4)n(C7H12O6) type, composed by a chain of polygallic esters (depside) linked, via ester bond, to one quinic acid.

\footnotetext{
a Instituto de Química-Física "Rocasolano”, CSIC, Serrano 119, 28006, Madrid, España. jdavalos@iqfr.csic.es

${ }^{\mathrm{b}}$ Facultad de Ingeniería Industrial y de Sistemas, Universidad Nacional Federico Villarreal, Av. Colonial 450, Lima 01, Perú.

${ }^{\text {c }}$ Facultad de Ing. Química, Universidad Nacional de Ingeniería, Av. Túpac Amaru s/n, Lima 01, Perú.

${ }^{\text {d }}$ Facultad de Ciencias, Universidad Nacional de Ingeniería, Av. Túpac Amaru s/n, Lima 01, Perú.
} 
Key words: Caesalpinia spinosa, commercial tara powder, hydrolysable tannins, MALDI/ FT-ICR, DFT/M05-2X theory, polygalloyquinic acids, gallic depsides.

\section{INTRODUCCIÓN}

La tara (Caesalpinia spinosa) es un árbol oriundo del Perú, país con mayor área de bosques naturales y mayor productor (aprox. 80\%) de tara en el mundo. La tara está distribuida, básicamente, en zonas semiáridas de los departamentos de Ayacucho, Cajamarca, La Libertad, Huancavelica, Apurímac, Ancash y Huánuco.

La tara, como fuente natural integral (tallo, hojas y frutos) es utilizada desde la época precolombina en la medicina popular como astringente, cicatrizante, antidisentérico y también como mordiente en tejidos. Sus propiedades benéficas se atribuyen a la gran capacidad antioxidante de sus taninos (compuestos polifenólicos). Estos pueden ser "condensados" o "hidrolizables"1,2. La tara en polvo contiene gran cantidad de estos últimos. La transformación de los taninos de la tara en compuestos fenólicos antioxidantes de bajo peso molecular constituye una excelente alternativa para la obtención de extractos de alto valor agregado, cuya demanda es creciente por su i/ amplia actividad farmacológica (incluso en el tratamiento del $\mathrm{SIDA}^{3}$ ) y antibacteriana ${ }^{4}$, ii/ clarificadores del vino y sustitutos de la malta en la elaboración de la cerveza, iii/ utilidad en la industria alimentaria, como suplemento alimenticio y/o sustituto de antioxidantes sintéticos ${ }^{5}$, algunos de los cuales podrían ser cancerígenos ${ }^{6,7}$. Frente a estas sospechas, la búsqueda de antioxidantes, como los taninos y polifenoles u otros productos con propiedades interesantes, provenientes de productos naturales, se ha convertido en un campo de investigación en auge ${ }^{8-12}$.

\section{PARTE EXPERIMENTAL}

\section{Extracción de taninos hidrolizables de la tara en polvo}

Los taninos hidrolizables estudiados fueron directamente extraídos de vainas de tara seca, sin semilla y previamente pulverizada, provenientes del paraje Uchuyhuaycco - Tambillos del departamento de Ayacucho-Perú. La extracción se hizo mediante una disolución del 80\% (v/v) de acetona/agua, mantenida $24 \mathrm{~h}$ en un baño a $40 \mathrm{oC}$ y finalmente centrifugada a 1500 rpm durante cinco minutos.

\section{Espectrometría de masas de ultra alta resolución FT-ICR con fuente de ionización por MALDI}

Los experimentos se llevaron a cabo utilizando el módulo MALDI ("Matrix-Assisted Laser Desorption/ Ionization") del espectrómetro de masas de ultra-alta resolución FT-ICR ("Fourier Transform Ion Cyclotron Resonance Mass Spectrometry") 13 930-Varian/Agilent, dotado con campo magnético permanente de 7.0 T, generado por una bobina superconductora inmersa en He-líquido. La fuente ionización por MALDI incluye un láser ORION Nd:YAG (longitud de onda $=355 \mathrm{~nm}$ ). 
La espectrometría FT-ICR, está basada en el movimiento ciclotrónico de partículas cargadas en una celda de confinamiento iónico y es, dentro de las espectrometrías de masas, una de las de mayor resolución $\left(\mathrm{R} \approx 10^{6}\right)$ y sensitividad. El rango de masas detectado por nuestro espectrómetro está entre 300 y $10000 \mathrm{Da}$; opera bajo ultra-alto vacío ( 10-10 mBar) tanto en los modos catiónico como aniónico. Para este trabajo operamos en el modo catiónico. Las muestras $(\sim 0.5 \mu \mathrm{L})$ fueron depositadas sobre una matriz cristalizada de ácido 2,6-dihidroxibenzoico.

La separación e identificación de las especies detectadas se realizaron sin necesidad de utilizar técnicas cromatográficas o de separación previas utilizadas habitualmente con espectrómetros de masas convencionales, y esto debido a que FT-ICR es un espectrómetro de ultra alta precisión $(<2 \mathrm{ppm})$. Utilizamos picos conocidos de malto-oligosacáridos de cerveza comercial para calibrar los registros de masas $^{14}$.

\section{DETALLES COMPUTACIONALES}

La geometría de los confórmeros más significativos tanto de los ácidos quínico y gálico como de sus derivados galo-quínicos (identificados experimentalmente) fueron optimizadas usando el método del Funcional de Densidad (DFT) de Truhlar, M05-2X ${ }^{14}$ con bases 6-311++G(d,p) para las especies sencillas y 6-31G(d) para las más complejas. Todos los cálculos se realizaron utilizando el paquete Gaussian $09^{16}$. El nivel de la teoría empleado ha mostrado ser una de las más adecuadas para el estudio estructural y energético de especies fenólicas y polifenólicas ${ }^{17,18}$. Los cálculos computacionales realizados sirvieron para dar soporte a los argumentos que sostenemos en la discusión de los resultados obtenidos.

\section{RESULTADOS Y DISCUSIÓN}

Los parámetros MALDI/FT-ICR de detección fueron optimizados para registrar señales intensas y bien resueltas en el rango de 500 a 2000 Da (Fig. 1). Estas señales corresponden a series de picos, igualmente espaciados en $152 \mathrm{Da}$. Hay una serie principal con picos en $\mathrm{m} /$ $z=671,839,991,1143,1295,1447$ y 1599 Da y otra secundaria (de picos menos intensos) desplazada en 16 Da respecto de la anterior. Cada pico de la serie en realidad incluye una distribución isotópica de picos de una misma especie, tal como se muestra en los recuadros de ampliación de la figura 1.

El análisis riguroso de cada una de las distribuciones antes descritas nos permite la identificación molecular inequívoca de la especie detectada. Tanto la serie principal como la secundaria corresponden a estructuras oligoméricas del tipo $\left(\mathrm{C}_{7} \mathrm{H}_{12} \mathrm{O}_{6}\right)\left(\mathrm{C}_{7} \mathrm{H}_{4} \mathrm{O}_{4}\right) \mathrm{n}$ con $\mathrm{n}=3$, $4, \ldots, 9$ asociados a cationes de potasio y de sodio, respectivamente. La unidad másica que se repite es un residuo de ácido gálico $\left(\mathrm{C}_{7} \mathrm{H}_{4} \mathrm{O}_{4} ; \mathrm{M}=152 \mathrm{Da}\right)$, el que llegaría a formar ésteres arílicos o dépsidos de polifenoles gálicos (unidos entre sí por un enlace de éster) enlazados, igualmente a través de un enlace de éster, al ácido quínico. 


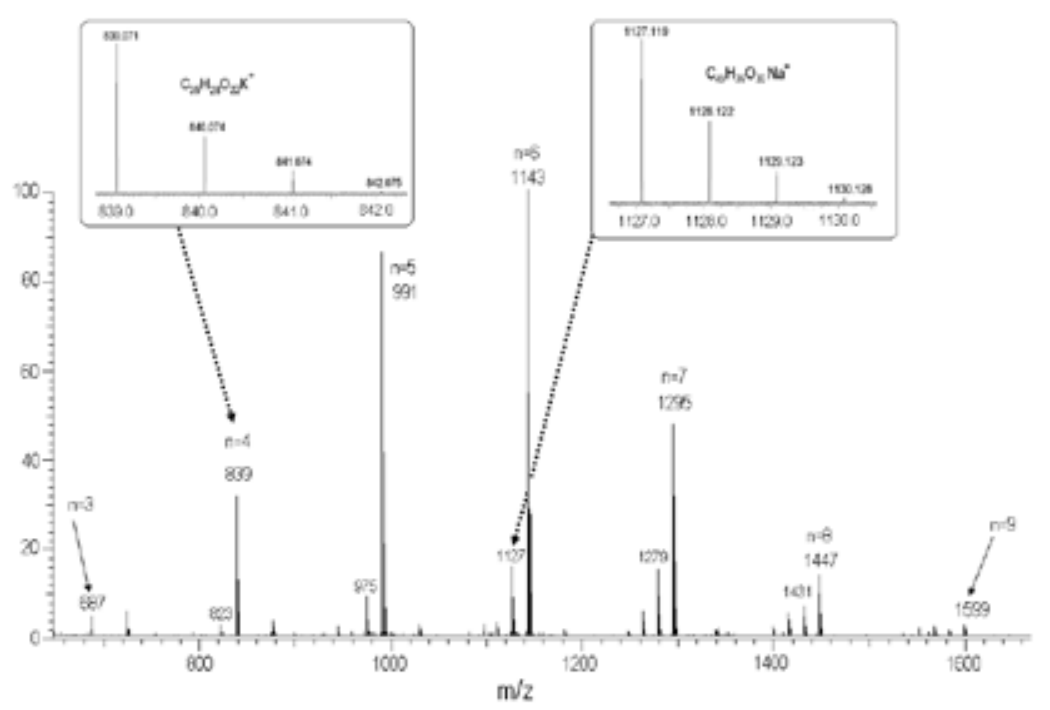

Figura 1. Espectro MALDI/FT-ICR, en modo positivo, de taninos hidrolizables extraídos de polvo de tara. Los cuadros superiores encierran señales ampliadas mostrando la distribución isotópica del pico $\mathrm{m} / \mathrm{z}$ identificado.

Pizzi et al. ${ }^{19}$ identificaron oligómeros similares a los que identificamos (entre $\mathrm{n}=1$ y 8 ) en tara hidrolizable comercial utilizando MALDI/TOF y HPLC. Sin embargo la estructura de los oligómeros que proponen (dépsidos gálicos unidos a un quínico) pese a ser sencilla y la más viable creemos que no está suficientemente fundamentada. Clifford et al. ${ }^{20}$ también identificaron estos oligómeros (entre $\mathrm{n}=1$ e incluso 9) extraídos de tara comercial metanolizada, utilizando LC-MS/ESI (cromatografía líquida y espectrometría de masas con fuente de electrospray o ESI). Las técnicas de fragmentación que utilizan para las especies detectadas les permiten proponer estructuras tentativas mejor elaboradas que las de Pizzi et $a l .{ }^{19}$ dado que incluyen la presencia de varios isómeros de cada especie detectada a los que identifican convenientemente como ácidos galo-quínicos. Estas estructuras son corroboradas por los resultados de Aouf et al. ${ }^{10}$ quienes identificaron, mediante UPLC (Ultraperformance Liquid Chromatography)-MS/ESI oligómeros entre $\mathrm{n}=1$ y 6 . Las estructuras propuestas por Clifford y Aouf se basan en aquellas reportadas por Haslam et al. ${ }^{21,22}$ y Horler-Nursten ${ }^{23}$ quienes, mediante técnicas de análisis cromatográfico, proponen que los oligómeros de ácidos galo-quínicos estarían constituidos no sólo por ácidos gálicos unidos, vía enlaces de éster, a los distintos sitios $\mathrm{OH}$ del ácido quínico, sino también por cadenas de dépsidos gálicos enlazados en sus posiciones meta y para.

En este contexto nosotros proponemos un análisis mecano-cuántico, usando la Teoría del Funcional de Densidad o DFT, para dilucidar la naturaleza de las especies identificadas partiendo de estructuras básicas como son los ácidos gálico $\left(\mathbf{G}, \mathrm{C}_{7} \mathrm{H}_{6} \mathrm{O}_{5}\right)$ y quínico $\left(\mathbf{Q}, \mathrm{C}_{7} \mathrm{H}_{12} \mathrm{O}_{6}\right)$ 
(figura 2). Estos son ácidos carboxílicos polifenólicos con sitios activos localizados en sus grupos $\mathrm{OH}$ y $\mathrm{COOH}$, capaces de formar enlaces de hidrógeno, enlaces de éster o desprotonarse con facilidad.

En $\mathbf{G}$, que es una molécula plana, el $\mathrm{OH}$ situado en la posición para (o C4) es el sitio más activo; mientras en $\mathbf{Q}$ es el grupo ácido $\mathrm{COOH}$. La orientación que adoptan los grupos $\mathrm{OH}$ define la naturaleza de sus correspondientes confórmeros (rotámeros). En la Fig. 2, se muestran los confórmeros $\mathbf{G}$ y $\mathbf{Q}$ más estables.

La reactividad de los centros activos los podemos cuantificar introduciendo el concepto energético de acidez intrínseca $(G A)$ de la especie considerada $\mathrm{AH}$ en fase gas, definido como el cambio de la energía de Gibbs de la siguiente reacción de transferencia protónica ${ }^{24}$ :

$$
\mathrm{AH} \rightarrow \mathrm{A}^{-}+\mathrm{H}^{+} \quad \Delta \mathrm{r} G^{0}(\mathrm{AH}, \mathrm{g})=G A
$$

donde $\mathrm{AH}$ es el ácido neutro y $\mathrm{A}^{-}$su anión producto de la desprotonación en un determinado sitio activo. Cuanto más bajos sean los valores de $G A$ cuanto más ácidos serán los correspondientes centros activos, de manera similar al significado del $\mathrm{pH}$ o del $\mathrm{pK}$ a de un ácido en disolución. Utilizando este concepto, determinamos que $\mathbf{Q}$ es más ácido en su grupo $\mathrm{COOH}(G A=1324 \mathrm{~kJ} / \mathrm{mol})$ que en el resto de sus grupos $\mathrm{OH}$; mientras que $\mathbf{G}$ lo es en su grupo $p$ - $\mathrm{OH}(\mathrm{GA}=1312 \mathrm{~kJ} / \mathrm{mol})$.

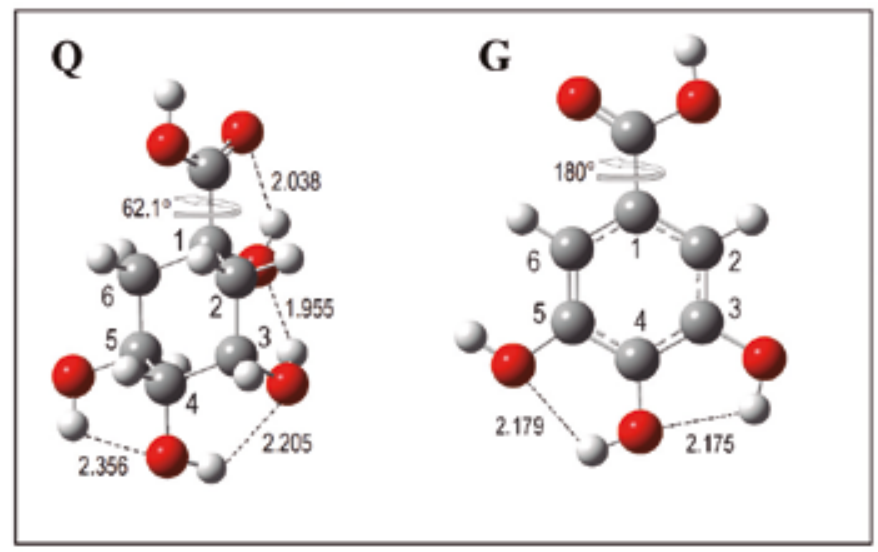

Figura 2. Estructura de los confórmeros más estables de los ácidos quínico (Q, izquierda) y gálico (G, derecha), ambas optimizadas al nivel M05-2X/6-311++G(d,p). Las distancias interatómicas están dadas en $\AA$. 
La estructura más sencilla del ácido gálico unido, vía enlace de éster, al ácido quínico (estructura galo-quínica $\mathbf{G}-\mathbf{Q}$, identificado por espectrometría de masas $\mathbf{M S}^{19,20}$ con $\mathbf{M}=344$ uma y $\mathrm{n}=1$ ) admite un considerable número de isómeros y confórmeros. Encontramos que la especie más estable incluye un enlace éster que une las posiciones meta $\mathrm{C}(5)$ de $\mathbf{G}$ y (C4) de $\mathbf{Q}$ (Fig. 3) y donde podemos apreciar también la presencia de un puente de $\mathrm{H}$ intermolecular entre el oxígeno más activo $\mathrm{O}(\mathrm{C} 4)$ de $\mathbf{G}$ y el $\mathrm{O}(\mathrm{C} 3)$ de $\mathbf{Q}$. Los isómeros $\mathbf{G}-\mathbf{Q}$ con enlaces de éster entre las posiciones $\mathrm{C} 1$ de $Q$ y otros sitios activos de $\mathbf{G}$ son considerablemente menos estables que el resto de combinaciones posibles, lo cual indica que la presencia de este tipo de isómeros (identificados por Clifford $^{20}$ como 1-O-galloyquinnic acid) son más bien irrelevantes.

Por otra parte, encontramos también computacionalmente que dépsidos de dos ácidos gálicos (G-G, éster di-gálico) se forman preferentemente uniendo, a través de un enlace éster, las posiciones meta $(\mathrm{C} 3)$ de un $\mathbf{G}$ con el grupo carboxílico del otro $\mathbf{G}$. Podemos apreciar en esta estructura la significativa presencia de un puente de $\mathrm{H}$ intermolecular entre el $\mathrm{OH}$ para y el oxígeno del carbonilo (Fig. 3-derecha).

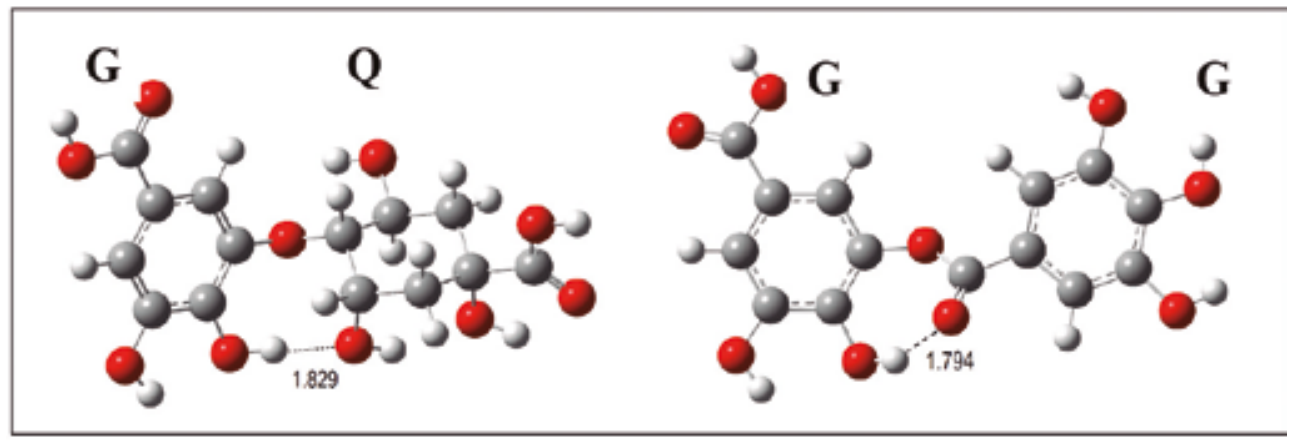

Figura 3. Estructura más estable de especies sencillas de ácido galo-quínico, G-Q,

(izquierda) y del dépsido gálico G-G, (derecha). Ambas estructuras fueron optimizadas al nivel M05-2X/6-31G(d). Las distancias interatómicas están dadas en $\AA$.

La estructura del oligómero galo-quínico Q-G-G identificado por $\mathrm{MS}^{19,20}$ con $\mathrm{M=} 496$ uma $\mathrm{y} \mathrm{n}=2$, tiene mayor número de combinaciones para formar isómeros y confórmeros que en el caso anterior. Encontramos que la estructura más estable está formada por un dépsido di-gálico G-G unida a un $\mathbf{Q}$ mediante un enlace éster que une las posiciones meta $\mathrm{C}(3)$ de $\mathbf{G}$ y (C5) de $\mathbf{Q}$. En esta estructura se puede apreciar la presencia de varios puentes de $\mathrm{H}$ (Fig. 4). Las otras combinaciones, particularmente aquellas que enlazan distintos sitios activos de $\mathbf{Q}$ son significativamente menos estables, por lo que deducimos que la presencia de estructuras identificados por Clifford ${ }^{20}$ como "1,X-di-O-digalloyquinnic acids" ( $\mathrm{X}=3,4$, 5) serían prácticamente irrelevantes. 


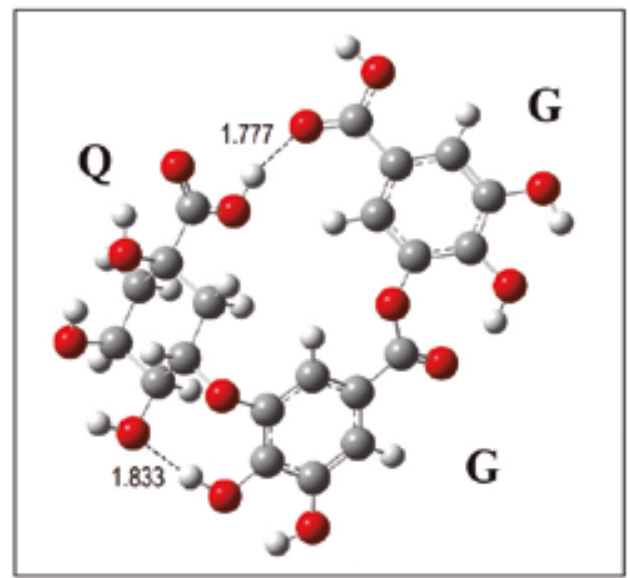

Figura 4. Estructura más estable del oligómero galo-quínico Q-G-G, formado por un dépsido di-gálico G-G enlazado a Q. Estructura optimizada al nivel M05-2X/6-31G(d). Las distancias interatómicas están dadas en $\AA$.

Teniendo en cuenta nuestro análisis computacional podemos deducir que los taninos hidrolizables de tara seca, identificados experimentalmente tanto por nosotros como por otros investigadores, tienen estructuras oligoméricas formadas principalmente por un dépsido gálico [cadena de Gs unidos entre sí por enlaces de éster entre las posiciones meta $\mathrm{C}(3)$ y $\mathrm{C}(\mathrm{OOH})$ ] enlazado también por un enlace éster al sitio meta (C5) del ácido quínico, tal como se muestra en la figura 5 .

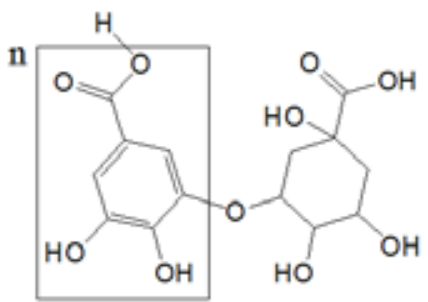

Figura 5. Estructura de taninos hidrolizables, de polvo de tara, formada por una cadena de dépsido n-gálico (recuadro) unido, vía enlace éster, al ácido quínico en su posición meta $\mathrm{C}(5)$.

\section{CONCLUSIONES}

El extracto de tara seca y pulverizada, obtenida por disolución agua/acetona, fue analizado mediante el espectrómetro de masas MALDI/FT-ICR de ultra-alta resolución (sin dispositivos de separación). Los resultados obtenidos, racionalizados por cálculos computacionales al nivel DFT/M05-2X, muestran que las especies identificadas son básicamente estructuras oligoméricas galo-quínicas, del tipo $\left(\mathrm{C}_{7} \mathrm{H}_{4} \mathrm{O}_{4}\right) \mathrm{n}\left(\mathrm{C}_{7} \mathrm{H}_{12} \mathrm{O}_{6}\right)$, formadas por una cadena de residuos de ésteres gálicos (dépsido) unida al ácido quínico. 


\section{AGRADECIMIENTO}

JZDP agradece al Programa PNICP (INNÓVATE PERÚ, ECIP-1-P-030-14) "Estancias cortas de investigadores peruanos residentes en el extranjero".

\section{REFERENCIAS BIBLIOGRÁFICAS}

1. Chambi F, Chirinos R, Pedreschi R, Betalleluz-Pallardel I, Debaste F, Campos D. Antioxidant potential of hydrolyzed polyphenolic extracts from C. spinosa (Caesalpinia spinosa) pods. Ind Crops Prod. 2013; 47: 168-175.

2. Grasel Fdos S, Ferrão MF, Wolf CR. Development of methodology for identification the nature of the polyphenolic extracts by FTIR associated with multivariate analysis. Spectrochim Acta A Mol Biomol Spectrosc. 2016;153:94-101.

3. Nishizawa M, Yamagishi T, Dutschman GE, Parker WB, Bodner AJ, Kilkuskie RE, et al. Anti-AIDS agents, 1. Isolation and characterization of four new tetragalloylquinic acids as a new class of HIV reverse transcriptase inhibitors from tannic acid. J Nat Prod. 1989;52(4):762-768.

4. Aguilar-Galvez A, Noratto G, Chambi F, Debaste F, Campos D. Potential of tara (Caesalpinia spinosa) gallotannins and hydrolysates as natural antibacterial compounds. Food Chem. 2014;156:301-304.

5. Skowyra M, Falguera V, Gallego G, Peiró S, Almajano MP. Antioxidant properties of aqueous and ethanolic extracts of tara (Caesalpinia spinosa) pods in vitro and in model food emulsions. J Sci Food Agric. 2014;94(5):911-918.

6. Hou DX. Potential mechanisms of cancer chemoprevention by anthocyanins. Curr Mol Med. 2003;3(2):149-159.

7. R. Prior. Absorption and metabolism of anthocyanins: potential health effects. En Meskin M, Bidlack W, Davies A, Lewis D, Randolph R. editores. Phytochemicals: mechanisms of action. Boca Raton, FL: CRC Press; 2004. p. 1-19.

8. Romero N, Fernández A, Robert P. A polyphenol extract of tara pods (Caesalpinia spinosa) as a potential antioxidant in oils. Eur J Lipid Sci Technol. 2012;114:951-957.

9. Urueña C, Mancipe J, Hernández J, Castañeda D, Pombo L, Gómez A, et al. Gallotanninrich Caesalpinia spinosa fraction decreases the primary tumor and factors associated with poor prognosis in a murine breast cancer model. BMC Complement Altern Med. 2013;13:74.

10. Aouf A, Benyahya S, Esnouf A, Caillol S, Boutevin B, Fulcrand H. Tara tannins as phenolic precursors of thermosetting epoxy resins. Eur Polym J. 2014; 55: 186-198.

11. He DY, Li YP, Tang HB, Luo L, Ma RJ, Wang JH, Wang LQ. Phenolic compounds from the twigs and leaves of Tara (Caesalpinia spinosa). J Asian Nat Prod Res. 2016;18(4):334-338.

12. Skowyra M, Janiewicz U, Salejda AM, Krasnowska G, Almajano MP. Effect of Tara (Caesalpinia spinosa) Pod Powder on the Oxidation and Colour Stability of Pork Meat Batter During Chilled Storage. Food Technol Biotechnol. 2015;53(4):419-427. 
13. Dávalos JZ, Guerrero A, Herrero R, Jiménez P, Abboud JLM. Espectrometría de Resonancia Ciclotrónica de Iones (FT-ICR): Una poderosa herramienta para el análisis estructural y el estudio de reacciones ión-molécula de una amplia variedad de especies. REVCIUNI. 2008; 12: 25.

14. Clowers BH, Dodds ED, Seipert RR, Lebrilla CB. Dual polarity accurate mass calibration for electrospray ionization and matrix-assisted laser desorption/ionization mass spectrometry using maltooligosaccharides. Anal Biochem. 2008;381(2):205-13.

15. Zhao JY, Schultz NE, Truhlar DG. Design of Density Functionals by Combining the Method of Constraint Satisfaction with Parametrization for Thermochemistry, Thermochemical Kinetics, and Noncovalent Interactions. J Chem Theory Comput. 2006; 2: 364-382.

16. Frisch MJ, Trucks GW, Schlegel HB, Scuseria GE, Robb MA, Cheeseman JR, et al. Gaussian 09, revision A.02. Wallingford, CT: Gaussian, Inc.; 2009.

17. Dávalos JZ, Herrero R, Costa JCS, Santos LMNBF, Liebman JF. Energetic and structural study of bisphenols. J Phys Chem A. 2014; 118: 3705-3709.

18. Dávalos JZ, Guerrero A, Valderrama-Negrón AC, Romero V, Lago AF. Energetics and structural properties of neutral and deprotonated phenyl carbinols. J Chem Thermodyn. 2016; 97: 315-321.

19. Giovando S, Pizzi A, Pasch H, Pretorius N. Structure and oligomers distribution of comercial tara (Caesalpina spinosa) hydrosable tannin. PRO LIGNO. 2013; 9: 22-31.

20. Clifford MN, Stoupi S, Kuhnert N. Profiling and characterization by LC-MSn of the galloylquinic acids of green tea, tara tannin, and tannic acid. J Agric Food Chem. 2007;55(8):2797-807.

21. Haslam E, Armitage R, Bayliss GS, Gramshaw JW, Haworth RD, Rogers HJ, et al. Gallotannins. Part III. The constitution of Chinese, Turkish, sumach, and tara tannins. J Chem Soc. 1961, 1842-1853.

22. Haslam E, Haworth RD, Keen PC. Gallotannins. Part VII. Tara gallotannin. J Chem Soc. 1962; 3814-3818.

23. Horler DF, Nursten HE. The tannins of tara, Caesalpina spinosa (mol.) kuntze. J Chem Soc. 1961, 3786-3792.

24. Guerrero A, Baer T, Chana A, González J, Dávalos JZ. Gas Phase Acidity Measurement of Local Acidic Groups in Multifunctional Species: Controlling the Binding Sites in Hydroxycinnamic Acids. J Am Chem Soc. 2013; 135: 9681-9690. 